\title{
Primary cyclic matrices in irreducible matrix subalgebras
}

\author{
Brian P. Corr and Cheryl E. Praeger \\ Communicated by Timothy C. Burness
}

\begin{abstract}
Primary cyclic matrices were used (but not named) by Holt and Rees in their version of Parker's MEAT-AXE algorithm to test irreducibility of finite matrix groups and algebras. They are matrices $X$ with at least one cyclic component in the primary decomposition of the underlying vector space as an $X$-module. Let $\mathrm{M}\left(c, q^{b}\right)$ be an irreducible subalgebra of $\mathrm{M}(n, q)$, where $n=b c>c$. We prove a generalisation of the Kung-Stong cycle index theorem, and use it to obtain a lower bound for the proportion of primary cyclic matrices in $\mathrm{M}\left(c, q^{b}\right)$. This extends work of Glasby and the second author on the case $b=1$.
\end{abstract}

\section{Introduction}

In order to improve and generalise the MEAT-AXE algorithm of Richard Parker [14], Holt and Rees [10] suggested the use of a family of matrices defined as follows: an $n \times n$ matrix $X$ over a field $F=\mathrm{GF}(q)$ is primary cyclic if, for some irreducible polynomial $f$ over $F$, the nullspace of $f(X)$ in $V(n, q)=F^{n}$ is an irreducible $F X$-submodule (see also Definition 2.3).

Given a group $G \leqslant \operatorname{GL}(n, F)$ acting on $V=F^{n}$, the irreducibility test in the MEAT-AXE algorithm, originally due to Simon Norton, tests whether or not $G$ leaves invariant a proper nontrivial subspace of $V$. The version of the test used by Holt and Rees in [10] does so by randomly searching for primary cyclic matrices and analysing their actions on $V$ : for the analysis it is crucial to know how abundant primary cyclic matrices are.

Holt and Rees in [10, pp.7-8] obtain a positive constant lower bound on the proportion of primary cyclic matrices in the full matrix algebra $\mathrm{M}(n, F)$, and in [7] Glasby and the second author show that the proportion of primary cyclic matrices in $\mathrm{M}(n, F)$ lies in the interval $\left(1-\frac{c_{1}}{q^{n}}, 1-\frac{c_{2}}{q^{n}}\right)$ for positive constants $c_{1}, c_{2}$. Here we focus on irreducible proper subalgebras of $\mathrm{M}(n, F)$ : any such subalgebra can be identified with the full matrix algebra $\mathrm{M}(c, K)$ over some extension field

The first author was supported by an Australian Postgraduate Award and UWA Top-Up Scholarship. This research forms part of Australian Research Council project DP110101153. 
$K=\mathrm{GF}\left(q^{b}\right)$, where $n=b c$ (see Section 2). We prove an analogous result to the Holt-Rees estimate for these subalgebras.

We treat the case of fixed degree extensions $\operatorname{GF}\left(q^{b}\right)$ of a field of fixed size $q$ as the dimension $n=b c$ grows unboundedly. Let $P_{M}\left(c, q^{b}\right)$ be the proportion of matrices in $\mathrm{M}\left(c, q^{b}\right)$ which are primary cyclic in $\mathrm{M}(n, q)$ relative to some irreducible polynomial $f$ of degree $b$ over $F$ (the minimal possible degree of such an $f$ ): then $P_{M}\left(c, q^{b}\right)$ is a lower bound for the proportion of primary cyclic matrices in $\mathrm{M}\left(c, q^{b}\right)$.

Theorem 1.1. Let $q$ be a prime power, and $b, c$ positive integers with $b>1$. Then

(i) $\lim _{c \rightarrow \infty} P_{M}\left(c, q^{b}\right)$ exists and equals

$P_{M}\left(\infty, q^{b}\right):=\lim _{c \rightarrow \infty} P_{M}\left(c, q^{b}\right)=1-\left(1-\frac{b q^{-b}}{\left(1-q^{-b}\right)^{2}} \omega\left(1, q^{b}\right)^{b}\right)^{N(q, b)}$

where $\omega\left(1, q^{b}\right)=\prod_{i=1}^{\infty}\left(1-q^{-b i}\right)$ and $N(q, b)$ is the number of monic irreducible polynomials of degree $b$ over $F_{q}$; and

(ii) there exists a constant $k(q, b)$ such that, if $c \geq\left(\frac{\max \left\{b-1, q^{b} / b\right\}}{\log (3 / 4)}\right)^{2}$, then

$$
\left|P_{M}\left(c, q^{b}\right)-P_{M}\left(\infty, q^{b}\right)\right|<k(q, b) q^{-b c} .
$$

Remark 1.2. (i) To prove Theorem 1.1, we use generating functions and in particular, we obtain a new generalisation in Theorem 3.6 of the Kung-Stong cycle index theorem (see $[11,16])$.

(ii) Theorem 1.1 shows that, for fixed $q, b$, the quantity $P_{M}\left(c, q^{b}\right)$ approaches its limiting value exponentially quickly. However the expression for the limit is rather complicated. We study the behaviour of the limiting value as $q^{b}$ grows, and prove (in Proposition 5.5) that the limit as $q^{b}$ approaches infinity of $P_{M}\left(\infty, q^{b}\right)$ exists and equals

$$
\lim _{q^{b \rightarrow \infty}} P_{M}\left(\infty, q^{b}\right)=1-e^{-1} .
$$

This is analogous to the original Holt-Rees estimate in [10] for the case $b=1$.

(iii) We prove Theorem 1.1 (ii) with the following value for the quantity $k(q, b)$ :

$$
k(q, b)=\frac{8}{3\left(1-q^{-b}\right)}\left(\frac{b q^{b}}{q^{b}-1} 2^{2 b} q^{2 b^{2}}\right)^{q^{b} / b}
$$

(see Proposition 5.10). We believe that this may be far from the best value.

(iv) A different subfamily of primary cyclic matrices was studied in [3], namely the set of all $f$-primary cyclic matrices $X \in \mathrm{M}\left(c, q^{b}\right)$ for irreducible polynomials 
$f$ of degree strictly greater than half the rank of $X$. In [3, Theorem 1.4], an explicit lower bound is given for the proportion of such primary cyclic matrices in $\mathrm{M}\left(c, q^{b}\right)$ as a function of $q, b$ and $c$. For large $b, c$ the bound is close to $\log _{e} 2$.

In Section 2 we present essential results on minimal and characteristic polynomials. In Section 3 we prove the generalisation of the Kung-Stong cycle index theorem and apply it to estimating the proportion of primary cyclic matrices in $\mathrm{M}\left(c, q^{b}\right)$. Section 4 deals with asymptotics and introduces a generating function crucial for the proof of Theorem 1.1. Then in Section 5 we complete the proof of Theorem 1.1, and discuss how to use it.

A consequence of Theorem 1.1 is that, for sufficiently large $c$, an explicit lower bound on the proportion of primary cyclic matrices can be calculated. Computationally we determine the proportion exactly for small $c$, see for example, Table 1: combining these two methods, we may address all values of $n$, so long as the field size $q^{b}$ is bounded.

\section{Preliminaries}

We first introduce some notation. Let $F$ be a field of order $q$ and let $K$ be an extension field of $F$ of degree $b$. The Galois group $G=\operatorname{Gal}(K / F) \leqslant$ Aut $K$ is cyclic of order $b$, generated by the Frobenius automorphism $\sigma_{0}: x \mapsto x^{q}$, and has the subfield $F$ as its fixed point set.

Let $V=F^{n}$ denote the space of $n$-dimensional row vectors over $F$, with standard basis $\left\{e_{1}, \ldots, e_{n}\right\}$, and let $\mathrm{M}(n, q)$ denote the full endomorphism ring of $V$, with elements written as $n \times n$ matrices with entries in $F$ relative to the standard basis. For a divisor $b$ of $n$ (say $n=b c$ ), we can embed the algebra $\operatorname{M}\left(c, q^{b}\right)$ as an irreducible subalgebra of $\mathrm{M}(n, q)$ as follows. The extension field $K$ is an $F$-vector space of dimension $b$, having as a basis $\left\{1, \omega, \omega^{2}, \ldots, \omega^{b-1}\right\}$, where $\omega$ is a primitive element of $K$. If $\left\{v_{1}, \ldots, v_{n}\right\}$ is a basis for $V\left(c, q^{b}\right)=K^{c}$, then $\left\{\omega^{i} v_{j} \mid 0 \leq i \leq b-1,1 \leq j \leq c\right\}$ is an $F$-basis for $V\left(c, q^{b}\right)$ as an $n$-dimensional $F$-vector space, where $n=b c$, and the mapping $\varphi: \omega^{i} v_{j} \mapsto e_{(j-1) b+i+1}$ extends linearly to an $F$-vector space isomorphism from $V\left(c, q^{b}\right)=K^{c}$ to $V$.

Each $X \in \mathrm{M}\left(c, q^{b}\right)$ defines an $F$-endomorphism of $V(c, K)$, and so we have an action of $\mathrm{M}\left(c, q^{b}\right)$ on $V=F^{n}$ defined by

$$
\text { (v) } X^{\varphi}:=v \varphi^{-1} X \varphi,
$$

for $v \in V$. Thus $X \mapsto X^{\varphi}$ defines an $F$-algebra monomorphism $\mathrm{M}\left(c, q^{b}\right) \rightarrow$ $\mathrm{M}(n, q)$, and we may identify $\mathrm{M}(c, K)$ with its image. This image is an irreducible $F$-subalgebra of $\mathrm{M}(n, q)$, and each irreducible subalgebra arises in this way (by Schur's lemma, see for example [4]). 
Throughout we will have to consider interchangeably the actions of a matrix in $\mathrm{M}\left(c, q^{b}\right)$ on two vector spaces, $F^{n}$ and $K^{c}$. For this reason we introduce notation to help keep track of which field we are dealing with.

Notation 2.1. $\quad$ (i) Let $V$ be the vector space $K^{c}$ of $c$-dimensional row vectors over $K=\mathrm{GF}\left(q^{b}\right)$, with $n=b c$. Then, as an $F$-vector space, $V$ is isomorphic, via $\varphi$ as defined above, to the vector space $F^{n}$. We denote this $F$ vector space by $V_{F}$. If there is any ambiguity we use $V_{K}$ to denote the $K$-vector space $V$. An element $X$ of $M\left(c, q^{b}\right)$ thus acts as a linear transformation of $V_{F}$ in a natural way (via the maps above): again we use the notation $X_{F}$ to denote the action of $X$ on $V_{F}$ (and similarly $X_{K}$ to denote the action on $V_{K}$ if there may be ambiguity).

(ii) We denote by $F[t], \operatorname{Irr}(q)$ and $\operatorname{Irr}(q, d)$ (where $d \geq 0$ ) the ring of polynomials over $F$, the set of monic irreducible polynomials over $F$, and the set of monic irreducibles of degree $d$ over $F$, respectively. Let $N(q, d)=$ $|\operatorname{Irr}(q, d)|$. Denote the characteristic and minimal polynomials of $X_{F}$ by $c_{X, F}(t), m_{X, F}(t)$, respectively, and similarly define $K[t], \operatorname{Irr}\left(q^{b}, d\right)$, $N\left(q^{b}, d\right)$ and $c_{X, K}(t), m_{X, K}(t)$ for the $X$-action on $V_{K}=K^{c}$.

(iii) The Galois group $G=\operatorname{Gal}(K / F)$ acts faithfully on $K[t]$ and $\mathrm{M}\left(c, q^{b}\right)$ by acting on the coefficients of a polynomial and the entries of a matrix, respectively. The fixed points of $G$ in these actions are respectively $F[t]$ and $\mathrm{M}(c, q)$.

(iv) If $U$ is an $X$-invariant $F$-subspace of $V$, then we denote by $\left.X\right|_{U}$ the restriction of $X$ to $U$; if in addition $U$ is a $K$-subspace, then we may write $\left(\left.X\right|_{U}\right)_{F}$ and $\left(\left.X\right|_{U}\right)_{K}$ if we wish to emphasise the field.

Definition 2.2. Let $X \in \mathrm{M}(n, q)$ and let $m_{X, F}=\prod_{i=1}^{r} f_{i}^{\alpha_{i}}$, with each $f_{i} \in \operatorname{Irr}(q)$ and $\alpha_{i}>0$. A useful $X$-invariant decomposition of $V_{F}$ is the $X$-primary decomposition (see [9, Theorem 11.8])

$$
V_{F}=V_{f_{1}} \oplus \cdots \oplus V_{f_{r}},
$$

where the subspace $V_{f_{i}}$ is called the $f_{i}$-primary component of $X$ (on $V$ ), and has the property that $f_{i}$ does not divide the minimal polynomial of the restriction of $X$ to $\bigoplus_{j \neq i} V_{f_{j}}$, and the minimal polynomial of $\left.X\right|_{V_{f_{j}}}$ is $f_{i}^{\alpha_{i}}$. Let

$$
\operatorname{Div}_{F}(X):=\left\{f_{1}, \ldots, f_{k}\right\} .
$$

If $f \in \operatorname{Irr}(q) \backslash \operatorname{Div}_{F}(X)$, we say that the $f$-primary component is trivial and define $V_{f}=\{0\}$. 
We also define $\operatorname{Div}_{K}(X)$ and the $X_{K}$-primary decomposition of $V_{K}$ similarly.

Definition 2.3. Let $X \in \mathrm{M}(n, q)$ and $f \in \operatorname{Irr}(q)$. Then $X$ is called $f$-primary cyclic if $\left.X\right|_{V_{f}}$ is nontrivial and cyclic. Also, $X$ is called cyclic if $X$ is $f$-primary cyclic for all $f \in \operatorname{Div}_{F}(X)$, or equivalently, if $m_{X, F}=c_{X, F}$. We also say that $X$ is primary cyclic if it is $f$-primary cyclic for some $f \in \operatorname{Irr}(q)$. We note that $X$ is $f$-primary cyclic if and only if the nullspace Null $f(X)$ is an irreducible $F X$-submodule of $V$.

\subsection{Minimal and characteristic polynomials}

We aim to count matrices $X$ in the subalgebra $\mathrm{M}\left(c, q^{b}\right)$ of $\mathrm{M}(n, q)$ such that $X_{F}$ is primary cyclic. To do so we derive necessary and sufficient conditions for this property which are intrinsic to their action on $K^{c}$ : that is to say, conditions on $X_{K}$. Our analysis follows that of [13, Section 5].

We investigate the relationship between the characteristic and minimal polynomials of a matrix $X$ over the two different fields $F$ and $K$. We call two polynomials $g, g^{\prime}$ in $K[t]$ conjugate if there exists $\sigma \in G=\operatorname{Gal}(K / F)$ such that $g^{\sigma}=g^{\prime}$. Recall Notation 2.1.

Lemma 2.4. Let $f \in \operatorname{Irr}(q, d)$, let $b \geq 2$, and let $G=\left\langle\sigma_{0}\right\rangle=\operatorname{Gal}(K / F)$. Suppose that $g \in \operatorname{Irr}\left(q^{b}\right)$ is a divisor of $f$ in $K[t]$. Then the following hold:

(i) $\operatorname{deg} g=d / \operatorname{gcd}(b, d)$;

(ii) $f=\operatorname{lcm}\left\{g^{\sigma_{0}^{i-1}} \mid 1 \leq i \leq b\right\}=\prod_{i=1}^{\mathrm{gcd}(b, d)} \mathrm{g}^{\sigma^{i-1}}$;

(iii) $g=g^{\sigma_{0}^{i}}$ if and only if $i \equiv 0(\bmod \operatorname{gcd}(b, d))$;

(iv) $f$ is the unique element of $\operatorname{Irr}(q)$ divisible by $g$ in $K[t]$.

Proof. Part (i) follows immediately from [12, Theorem 3.46]. For (ii) and (iii), observe that since $\sigma_{0}$ fixes the field $F$, the image $g^{\sigma_{0}}$ divides $f^{\sigma_{0}}=f$, and similarly, for every $i$ we have $g^{\sigma_{0}^{i}} \mid f$, so

$$
\operatorname{lcm}\left\{g^{\sigma_{0}^{i-1}} \mid 1 \leq i \leq b\right\} \text { divides } f .
$$

Since the set $\left\{g^{\sigma_{0}^{i-1}} \mid 1 \leq i \leq b\right\}$ is permuted under the action of $\sigma_{0}$, its least common multiple is fixed by $\sigma_{0}$, and so lies in $F[t]$. Then by the irreducibility of $f$, they are equal.

Since $\operatorname{deg} f=d=\operatorname{gcd}(b, d) \operatorname{deg} g$, it follows that $\left\{g^{\sigma_{0}^{i-1}} \mid 1 \leq i \leq b\right\}$ has size $\operatorname{gcd}(b, d)$, and the stabiliser of each $g^{\sigma_{0}^{i-1}}$ in $G$ is $\left\langle\sigma_{0}^{\operatorname{gcd}(b, d)}\right\rangle$. This implies part (iii) and the last assertion of (ii). Part (iv) follows from part (ii). 
We now give a description of $f$-primary cyclic matrices in terms of their representations over the field $K$. The following result is derived from [13, Lemma 5.1 and Corollary 5.2]. Recall the notation for minimal polynomials from Notation 2.1.

Proposition 2.5. Let $f \in \operatorname{Irr}(q, d)$, let $G=\operatorname{Gal}(K / F)$, and let $X \in \mathrm{M}\left(c, q^{b}\right)$ such that $f \in \operatorname{Div}_{F}(X)$. Then $X_{F}$ is $f$-primary cyclic if and only if $b \mid d$ and the following hold for some irreducible divisor $g \in K[t]$ of $f$ of degree $d / b$ :

(i) $g \in \operatorname{Div}_{K}(X)$ and $X_{K}$ is $g$-primary cyclic; and

(ii) for each nontrivial $\sigma \in G$, the conjugate $g^{\sigma} \neq g$ and $g^{\sigma} \notin \operatorname{Div}_{K}(X)$.

Proof. By [13, Lemma 5.1],

$$
m_{X, F}=\operatorname{lcm}\left\{m_{X, K}^{\sigma} \mid \sigma \in G\right\} .
$$

If $g \in \operatorname{Irr}\left(q^{b}\right)$ divides $f$, then, by Lemma 2.4 (ii), $f$ is the product of the distinct conjugates of $g$ by elements of $G$. Since $f \mid m$, it follows from (2.1) that $m_{X, K}$ is divisible by at least one conjugate of $g$. Without loss of generality $g \mid m_{X, K}$. Note that, by Lemma 2.4 (ii), $f=\operatorname{lcm}\left\{g^{\sigma} \mid \sigma \in G\right\}$. Consider the following $X_{K^{-}}$ invariant decomposition of $V=V_{K}$ :

$$
V_{K}=V_{0} \oplus V_{1},
$$

where $V_{0}$ is the sum of the $g^{\sigma}$-primary components of $V$, for $\sigma \in G$, and $V_{1}$ is the sum of the other primary components. Let $Y_{i}=\left.X\right|_{V_{i}}$ for $i=0$, 1 . Then $g \mid m_{Y_{0}, K}$, and the only irreducible divisors of $m_{Y_{0}, K}$ are $g^{\sigma}$ for certain $\sigma \in G$. Also $m_{Y_{1}, K}$ is coprime to $\operatorname{lcm}\left\{g^{\sigma} \mid \sigma \in G\right\}=f$. By [13, Lemma 5.1] applied to $Y_{0}$ acting on $V_{0}$, we have $m_{Y_{0}, F}=\operatorname{lcm}\left\{m_{Y_{0}, K}^{\sigma} \mid \sigma \in G\right\}$, and it follows from Lemma 2.4 (iv), that $f$ is the only irreducible divisor of $m_{Y_{0}, F}$. Thus $V_{0}$ is the $f$-primary component of $V_{F}$.

By definition, $X_{F}$ is $f$-primary cyclic if and only if $\left(Y_{0}\right)_{F}$ is cyclic (hence with minimum polynomial $m_{Y_{0}, F}=f$ ). By [13, Corollary 5.2] applied to $Y_{0}$, this holds if and only if (i) $\left(Y_{0}\right)_{K}$ is cyclic, and (ii) $m_{Y_{0}, K}$ is coprime with $m_{Y_{0}, K}^{\sigma}$ for all nontrivial $\sigma \in G$. Recall that $g \mid m_{Y_{0}, K}$ and $g^{\sigma} \nmid m_{Y_{1}, K}$ for all $\sigma \in G$. Thus condition (ii) is equivalent to (ii) ${ }^{\prime} m_{Y_{0}, K}=g^{k}$ for some positive integer $k$ and, for all nontrivial $\sigma \in G, g^{\sigma} \neq g$ and $g^{\sigma} \nmid m_{X, K}$. The first assertion of (ii) ${ }^{\prime}$ holds if and only if $V_{0}$ is the $g$-primary component of $X_{K}$. By Lemma 2.4 (iii), the second assertion in (ii)' holds if and only if $b \mid d$, $\operatorname{deg} g=d / b$, and $g^{\sigma} \nmid m_{X, K}$ for all nontrivial $\sigma \in G$. Also if $\left(Y_{0}\right)_{K}$ is cyclic with minimal polynomial $g^{k}$, then $k$ must be 1 . Thus both of the conditions (i) and (ii) hold if and only if $b \mid d$, $\operatorname{deg} g=d / b, X_{K}$ is $g$-primary cyclic, and $g^{\sigma} \neq g$ and $g^{\sigma} \nmid c_{X, K}$ for all nontrivial $\sigma \in G$ (recalling that $m_{X, K}$ and $c_{X, K}$ have the same set of irreducible divisors.) 
The next corollary follows immediately from Lemmas 2.5 and 2.4 (iii).

Corollary 2.6. Let $X \in \mathrm{M}\left(c, q^{b}\right) \subseteq \mathrm{M}(n, q)$, where $n=b c$, let $G=\operatorname{Gal}(K / F)$, and let $I=\left\{f_{1}, \ldots, f_{k}\right\} \subset \operatorname{Irr}(q, b)$. Then $X_{F}$ is $f_{i}$-primary cyclic, for every $i \leq k$, if and only if there exists a set $I^{\prime}=\left\{g_{1}, \ldots, g_{k}\right\} \subseteq \operatorname{Irr}\left(q^{b}, 1\right)$ with $\left|I^{\prime}\right|=k$ satisfying the following for each $i \in\{1, \ldots, k\}$ :

(i) $g_{i} \mid f_{i}$, and $X_{K}$ is $g_{i}$-primary cyclic;

(ii) for each nontrivial $\sigma \in G$, we have $g_{i}^{\sigma} \neq g_{i}$, and $g_{i}^{\sigma} \notin \operatorname{Div}_{K}(X)$.

\section{A generalised cycle index for matrix algebras}

Our main tool in enumerating matrices is the cycle index of the matrix algebra $\mathrm{M}(n, q)$, introduced by Kung [11] and developed further by Stong [16], and based on Polya's cycle index (see for example [15]) of a permutation group. We continue to use Notation 2.1. To each pair $(h, \lambda)$, with $h \in \operatorname{Irr}(q)$ and $\lambda$ a partition of a nonnegative integer, denoted $|\lambda|$, with $|\lambda| \in[0, n]$, assign an indeterminate $x_{h, \lambda}$. Then the cycle index of $\mathrm{M}(n, q)$ is the multivariate polynomial

$$
Z_{\mathrm{M}(n, q)}(\mathbf{x}):=\frac{1}{|\operatorname{GL}(n, q)|} \sum_{X \in \mathrm{M}(n, q)}\left(\prod_{h \in \operatorname{Div}_{F} X} x_{h, \lambda(X, h)}\right),
$$

where $\mathbf{x}$ is a vector representing the set of indeterminates $x_{h, \lambda}$ occurring, and $\lambda(X, h)$ is a partition (of an integer) uniquely determined by the structure of the action of $X$ on the primary component $V_{h}$ as described in Definition 3.1 below.

In this section we generalise the cycle index of Kung and Stong to include variables associated with a finite number of irreducible polynomials which do not necessarily divide $c_{X, F}(t)$. We will apply this more general version in our study of primary cyclic matrices. We begin by presenting the original cycle index theorem. In this section $V=F^{c}$ is viewed solely as an $F$-space, where, recall, $F=\operatorname{GF}(q)$.

Definition 3.1. Let $X \in \mathrm{M}(n, q), h \in \operatorname{Irr}(q)$, and let $\alpha_{h}$ be the multiplicity of $h$ in $c_{X, F}(t)$, so that $X$ acts on the $h$-primary component $V_{h}$ of $V_{F}$ with characteristic polynomial $h^{\alpha_{h}}$ and $\alpha_{h} \operatorname{deg} h=\operatorname{dim}\left(V_{h}\right)_{F}$. (In particular, $\alpha_{h}=0$ if $V_{h}=0$.) There is a direct sum decomposition of $V_{h}$ into $F X$-submodules

$$
V_{h}=V_{\lambda_{1}} \oplus \cdots \oplus V_{\lambda_{r}}
$$

with each $V_{\lambda_{i}}$ cyclic, such that the restriction of $X$ to $V_{\lambda_{i}}$ has minimal polynomial $h^{\lambda_{i}}$, and $\lambda_{i} \geq \lambda_{i+1}$ for all $i$. The $\lambda_{i}$ are uniquely determined by $X$ (see [9, Theorem 11.19]). Define the partition $\lambda(X, h)$ as the sequence

$$
\lambda(X, h):=\left(\lambda_{1}, \lambda_{2}, \ldots, \lambda_{r}, 0,0, \ldots\right) .
$$


Then $\lambda(X, h)$ is a partition of $\alpha_{h}$, and as this partition is non-increasing, we often omit the 'trailing zeroes' and write $\left(\lambda_{1}, \ldots, \lambda_{r}\right)$ if $V_{h} \neq\{0\}$ and ()$:=(0,0, \ldots)$ (the empty partition of the integer zero) if $V_{h}=\{0\}$.

In particular, $\lambda(X, h)=()$ if $h \notin \operatorname{Div}_{F}(X)$, and otherwise $\lambda(X, h)$ is determined by the sizes of the blocks in the Frobenius normal form of $\left.X\right|_{V_{h}}$.

See [9] for more information on the cyclic and primary decompositions, and on $\lambda(X, h)$. Lemma 3.2 follows immediately from the definition of $\lambda(X, h)$ :

Lemma 3.2. Let $X \in \mathrm{M}(n, q), h \in \operatorname{Irr}(q)$, and $\lambda=\lambda(X, h)$. Then the following hold:

(i) $h \notin \operatorname{Div}_{F}(X)$ if and only if $\lambda(X, h)=()$;

(ii) $h \in \operatorname{Div}_{F}(X)$ and $X$ is $h$-primary cyclic if and only if $\lambda(X, h)=\left(\lambda_{1}\right)$, with $\lambda_{1}>0$, and in this case $\lambda_{1}$ is the multiplicity of $h$ in $c_{X, F}(t)$;

(iii) $h \in \operatorname{Div}_{F}(X)$ and $X$ is not h-primary cyclic if and only if $\lambda(X, h)$ has at least two nonzero parts.

Definition 3.3. Let $\lambda$ be a partition of an integer $|\lambda|$, let $h \in \operatorname{Irr}(q)$, and let $s=$ $|\lambda| \operatorname{deg} h$. If $\lambda=(), \operatorname{define} c(\lambda, \operatorname{deg} h, q)=1$. If $|\lambda| \geq 1$, then there exists a matrix $X:=X_{\lambda, h} \in \mathrm{M}(s, q)$ such that $c_{X, F}(t)=h^{|\lambda|}$, and the cyclic decomposition of $F^{s}$ described in Definition 3.1 determines the partition $\lambda$. In this case we define

$$
c(\lambda, \operatorname{deg} h, q):=\left|C_{\mathrm{GL}(s, q)}(X)\right| .
$$

Note that $c(\lambda, \operatorname{deg} h, q)$ (the number of matrices in $\operatorname{GL}(s, q)$ which commute with $X$ ) depends only on $\operatorname{deg} h$ and $\lambda$, since all matrices $X$ with these properties are conjugate under elements of GL(s,q) (see again [9, Theorem 11.19]). The number of such matrices $X$ is $|\mathrm{GL}(s, q)| / c(\lambda, \operatorname{deg} h, q)$, and this holds also for $\lambda=()$ if we take $\operatorname{GL}(0, q)$ as the trivial group. The Kung-Stong cycle index theorem is stated in terms of these quantities.

Theorem 3.4 (Cycle index theorem). The generating function for the cycle index of a matrix algebra $\mathrm{M}(n, q)$ satisfies

$$
1+\sum_{n=1}^{\infty} Z_{M(n, q)}(\mathbf{x}) u^{n}=\prod_{h \in \operatorname{Irr}(q)}\left(1+\sum_{\lambda \neq()} x_{h, \lambda(h)} \frac{u^{|\lambda| \operatorname{deg} h}}{c(\lambda, \operatorname{deg} h, q)}\right) .
$$

Theorem 3.4 assigns to each $X \in \mathrm{M}(n, q)$ a monomial $\prod_{h \in \operatorname{Div}_{F} X} x_{h, \lambda(X, h)}$, and sums over $\mathrm{M}(n, q)$. We generalise by forcing a certain finite collection of indeterminates to occur in the monomials for all matrices $X$, whether or not the 
corresponding irreducibles divide $c_{X, F}(t)$. The reason for this generalisation will become apparent when we apply this to the proof of Lemma 4.4 in Section 4: it permits us to ask questions about whether some (fixed) $f \in \operatorname{Irr}(q) \operatorname{divides} c_{X, F}(t)$.

Definition 3.5. For a finite subset $I \subset \operatorname{Irr}(q)$, and partitions $\lambda(X, h)$ as in Definition $3.1(X \in \mathrm{M}(n, q), h \in \operatorname{Irr}(q))$, the $I$-cycle index of $\mathrm{M}(n, q)$ is defined as

$$
Z_{\mathrm{M}(n, q)}^{(I)}(\mathbf{x}):=\frac{1}{|\mathrm{GL}(n, q)|} \sum_{X \in \mathrm{M}(n, q)}\left(\prod_{h \in \operatorname{Div}_{F}(X) \cup I} x_{h, \lambda(X, h)}\right),
$$

or equivalently

$$
\begin{aligned}
Z_{\mathrm{M}(n, q)}^{(I)}(\mathbf{x}):= & \frac{1}{|\operatorname{GL}(n, q)|} \\
& \times \sum_{X \in \mathrm{M}(n, q)}\left(\left(\prod_{h \in \operatorname{Div}_{F}(X)} x_{h, \lambda(X, h)}\right)\left(\prod_{h \in I \backslash \operatorname{Div}_{F}(X)} x_{h,()}\right)\right) .
\end{aligned}
$$

The Kung-Stong cycle index is precisely the $I$-cycle index with $I=\emptyset$. We now prove the $I$-cycle index theorem.

Theorem 3.6 ( $I$-cycle index theorem). For a finite subset $I \subseteq \operatorname{Irr}(q)$ and $\lambda(X, h)$, $c(\lambda, \operatorname{deg} h, q)$ as in Definitions 3.1 and 3.3, the generating function for the I-cycle index of $\mathrm{M}(n, q)$ satisfies

$$
\begin{aligned}
& \prod_{h \in I} x_{h,()}+\sum_{n=1}^{\infty} Z_{M(n, q)}^{(I)}(\mathbf{x}) u^{n}=\prod_{h \in \operatorname{Irr}\left(q^{b}\right) \backslash I}\left(1+\sum_{\lambda \neq()} x_{h, \lambda} \frac{u^{|\lambda| \operatorname{deg} h}}{c(\lambda, \operatorname{deg} h, q)}\right) \\
& \times \prod_{h \in I}\left(x_{h,()}+\sum_{\lambda \neq()} x_{h, \lambda} \frac{u^{|\lambda| \operatorname{deg} h}}{c(\lambda, \operatorname{deg} h, q)}\right) .
\end{aligned}
$$

Proof. Our proof follows that of Stong in [16]. We consider the quantities in (3.3) as power series in the variables $x_{h, \lambda}$, and treat $u$ as a constant. Note that since $I$ is finite, and for $X \in \mathrm{M}(n, q)$ the set $\operatorname{Div}_{F} X$ is finite, each $Z_{\mathrm{M}(n, q)}^{(I)}(\mathbf{x})$ on the left-hand side of (3.3), when expressed as in (3.2), is clearly a sum of products of finitely many of the $x_{h, \lambda}$. Recall that $c((), \operatorname{deg} h, q)=1$ for all $h \in \operatorname{Irr}(q)$, and so

$$
x_{h,()}=x_{h,()} \frac{u^{0 \cdot \operatorname{deg} h}}{c((), \operatorname{deg} h, q)} .
$$

Let $\left\{h_{i} \mid 1 \leq i \leq t\right\} \subseteq \operatorname{Irr}(q)$, and let $\left\{\lambda_{i} \mid 1 \leq i \leq t\right\}$ be a multiset of partitions such that $\lambda_{i}$ may be () if $h_{i} \in I$, and otherwise $\lambda_{i} \neq\left(\right.$ ). For each $i$, let $n_{i}=$ $\left|\lambda_{i}\right| \operatorname{deg} h_{i}$, and let $n=\sum_{i=1}^{t} n_{i}$. The coefficient of $\prod_{i=1}^{t} x_{h_{i}, \lambda_{i}}$ on the right-hand 
side of (3.3) is

$$
\left(\prod_{i=1}^{t} \frac{1}{c\left(\lambda_{i}, \operatorname{deg} h_{i}, q\right)}\right) u^{n} .
$$

On the other hand, the coefficient of $\prod_{i=1}^{n} x_{h_{i}, \lambda_{i}}$ on the left-hand side of (3.3) is equal to 1 if $n=0$, and otherwise is $u^{n} /|\operatorname{GL}(n, q)|$ times the number of matrices $X \in \mathrm{M}(n, q)$ having characteristic polynomial $\prod_{i=1}^{t} h_{i}^{\left|\lambda_{i}\right|}$, with $\lambda\left(X, h_{i}\right)=\lambda_{i}$ for each $i$. Each of these matrices $X$ is uniquely determined by the following data:

(i) its primary decomposition $V=V_{h_{1}} \oplus \cdots \oplus V_{h_{n}}$ is such that $\operatorname{dim} V_{h_{i}}=n_{i}$, noting that we may have $\lambda\left(X, h_{i}\right)=()$ if $h_{i} \in I$; and

(ii) for each primary component $V_{h_{i}}$, the partition $\lambda_{i}=\lambda\left(X_{h_{i}}, h_{i}\right)$.

There are exactly

$$
\frac{|\mathrm{GL}(n, q)|}{\prod_{i=1}^{n}\left|\mathrm{GL}\left(n_{i}, q\right)\right|}
$$

direct sum decompositions of $V$ with the appropriate dimensions, and on each of the parts $V_{h_{i}}$, there are exactly $\left|\operatorname{GL}\left(n_{i}, q\right)\right| / c\left(\lambda_{i}, \operatorname{deg} h_{i}, q\right)$ matrices $X_{h_{i}}$ such that $\lambda\left(X_{h_{i}}, h_{i}\right)=\lambda_{i}$, as noted in Definition 3.3. Thus the coefficient of $\prod_{i=1}^{t} x_{h_{i}, \lambda_{i}}$ on the left-hand side of (3.3) is

$$
\begin{aligned}
& \frac{u^{n}}{|\mathrm{GL}(n, q)|} \cdot \frac{|\mathrm{GL}(n, q)|}{\prod_{1 \leq i \leq t}\left|\mathrm{GL}\left(n_{i}, q\right)\right|} \cdot \prod_{1 \leq i \leq t} \frac{\left|\mathrm{GL}\left(n_{i}, q\right)\right|}{c\left(\lambda_{i}, \operatorname{deg} h_{i}, q\right)} \\
& \quad=\prod_{1 \leq i \leq t} \frac{1}{c\left(\lambda_{i}, \operatorname{deg} h_{i}, q\right)} u^{n}
\end{aligned}
$$

which equals (3.4).

\section{Counting}

By evaluating (3.3) in Theorem 3.6 at different values of $\mathbf{x}$, we can enumerate subsets of $\mathrm{M}\left(c, q^{b}\right)$ having certain properties based on their minimal polynomials. In particular, we wish to count matrices in $\mathrm{M}\left(c, q^{b}\right) \subseteq \mathrm{M}(n, q)$ which are $f$-primary cyclic for some $f \in \operatorname{Irr}(q, b)$ (recall that by Proposition $2.5, b$ is the smallest degree for which such $f$-primary matrices exist). We begin this section by introducing some quantities which will simplify our rather complicated calculations.

Note that while the $I$-cycle index theorem was presented for the full matrix algebra $\mathrm{M}(n, q)$, it may be applied directly to the irreducible subalgebra $\mathrm{M}\left(c, q^{b}\right)$, provided that we treat $\mathrm{M}\left(c, q^{b}\right)$ in its own right, rather than as a subalgebra of $\mathrm{M}(b c, q)$. 
Definition 4.1. Define the following quantities:

$$
\begin{array}{rlr}
\omega_{n}(u, q) & :=\prod_{i=1}^{n}\left(1-u q^{-i}\right) & \text { for }\{u \in \mathbb{C}:|u|<q\} ; \\
\omega(u, q):=\prod_{i=1}^{\infty}\left(1-u q^{-i}\right) & \text { for }\{u \in \mathbb{C}:|u|<q\} ; \\
G(u, q, n):=1+\sum_{\lambda \neq()} \frac{u^{|\lambda|}}{c(\lambda, n, q)} & \text { for }\{u \in \mathbb{C}:|u|<1\} ; \\
P(u, q):=1+\sum_{n=1}^{\infty} \frac{u^{n}}{\omega_{n}(1, q)} & \text { for }\{u \in \mathbb{C}:|u|<1\} ; \\
S(u, q):=\sum_{n=1}^{\infty} \frac{u^{n}}{q^{n}\left(1-q^{-1}\right)} & \text { for }\{u \in \mathbb{C}:|u|<q\} ;
\end{array}
$$

where $c(\lambda, n, q)$ is as in Definition 3.3. Note that

$$
\omega_{n}(1, q)=\frac{|\mathrm{GL}(n, q)|}{|\mathrm{M}(n, q)|},
$$

and that $\omega(1, q)=\lim _{n \rightarrow \infty}|\mathrm{GL}(n, q)| /|\mathrm{M}(n, q)|$ exists.

These definitions simplify our rather complicated calculations later. The following results will be used to manipulate the generating functions:

Lemma 4.2. The following relations hold between the quantities in Definition 4.1, for $|u|<1$, and in case (4.3) for $|u|<q$ :

$$
\begin{aligned}
& G(u, q, 1)=P\left(u q^{-1}, q\right) ; \\
& \prod_{h \in \operatorname{Irr}(q)} G\left(u^{\operatorname{deg} h}, q, \operatorname{deg} h\right)=P(u, q) ; \\
& P(u, q)=\frac{1}{1-u} P\left(u q^{-1}, q\right)=\prod_{i=0}^{\infty}\left(1-u q^{-i}\right)^{-1} ; \\
& S\left(u, q^{b}\right)=\frac{1}{\left(q^{b}-1\right)} \frac{u}{\left(1-u q^{-b}\right)} ;
\end{aligned}
$$

Proof. (4.1): In equation (3.3) set $I=\emptyset$, and for all $\lambda$, set $x_{h, \lambda}=0$ if $h \neq t-1$ and $x_{t-1, \lambda}=1$. Using (3.1), we see that the right-hand side of (3.3) is equal to 
$G(u, q, 1)$, while the left-hand side is

$$
1+\sum_{n=1}^{\infty} u^{n} \cdot\left(\frac{\text { \# unipotent elements in } \mathrm{M}(n, q)}{|\mathrm{GL}(n, q)|}\right)
$$

which by Steinberg's theorem [2, Theorem 6.6.1] is equal to

$$
1+\sum_{n=1}^{\infty} \frac{u^{n} q^{n(n-1)}}{|\operatorname{GL}(n, q)|}=P\left(u q^{-1}, q\right) .
$$

(4.2): The left-hand side of equation (4.2) is equal to the right-hand side of (3.3) if we set $I=\varnothing$ and all the $x_{h, \lambda}=1$. Thus by (3.3), using also (3.1) and Definition 4.1 , this is equal to

$$
1+\sum_{n=1}^{\infty} \frac{|\mathrm{M}(n, q)|}{|\mathrm{GL}(n, q)|} u^{n}=P(u, q) .
$$

(4.3): In [1, p. 19] we find the equality, for $|u|<q$,

$$
\prod_{r=1}^{\infty}\left(1-u q^{-r}\right)^{-1}=1+\sum_{n=1}^{\infty} \frac{u^{n} q^{n(n-1) / 2}}{\prod_{i=1}^{n}\left(q^{i}-1\right)},
$$

the right-hand side of which is equal to $P\left(u q^{-1}, q\right)$. This proves the second equality of (4.2), and the first equality follows on substituting $u$ for $u q^{-1}$ into the second equality.

(4.4): This is a routine geometric series calculation.

Definition 4.3. Noting that $\operatorname{Irr}(q, b)$ is a finite set,

(i) for a nonempty subset $I \subseteq \operatorname{Irr}(q, b)$, define $\operatorname{pcbI}\left(I, c, q^{b}\right):=\left\{X \in \mathrm{M}\left(c, q^{b}\right) \mid X_{F}\right.$ is $f$-primary cyclic for all $\left.f \in I\right\} ;$

(ii) define

$$
\operatorname{pcb}\left(c, q^{b}\right):=\bigcup_{\substack{I \subseteq \operatorname{Irr}(q, b) \\ I \neq \emptyset}} \operatorname{pcbI}\left(I, c, q^{b}\right) ;
$$

so $P_{M}\left(c, q^{b}\right)=\left|\operatorname{pcb}\left(c, q^{b}\right)\right| /\left|M\left(c, q^{b}\right)\right|$; 
(iii) for nonempty $I \subseteq \operatorname{Irr}(q, b)$, define generating functions for pcbI and pcb:

$$
\begin{gathered}
\operatorname{PCBI}\left(I, u, q^{b}\right):=1+\sum_{c=1}^{\infty} \frac{\left|\operatorname{pcbI}\left(I, c, q^{b}\right)\right|}{\left|\operatorname{GL}\left(c, q^{b}\right)\right|} u^{c}, \\
\operatorname{PCB}\left(u, q^{b}\right):=1+\sum_{c=1}^{\infty} \frac{\left|\operatorname{pcb}\left(c, q^{b}\right)\right|}{\left|\operatorname{GL}\left(c, q^{b}\right)\right|} u^{c} .
\end{gathered}
$$

Note that $\operatorname{pcb}\left(c, q^{b}\right)$ is the set of matrices $X \in \mathrm{M}\left(c, q^{b}\right)$ such that $X_{F}$ is $f$ primary cyclic for some $f \in \operatorname{Irr}(q, b)$ : hence the name 'primary cyclic, degree $\mathbf{b}$ '. Our end goal is to find and investigate $\operatorname{PCB}\left(u, q^{b}\right)$ : to do so we compute a formula for $\operatorname{PCBI}\left(I, u, q^{b}\right)$, depending only on the size of $I$ and the parameters $q, b$, and a relationship between the functions PCB, PCBI.

Lemma 4.4. Let $I=\left\{f_{1}, \ldots, f_{k}\right\} \subseteq \operatorname{Irr}(q, b)$, with $|I|=k$. Then for the generating function $\operatorname{PCBI}\left(I, u, q^{b}\right)$ as in Definition 4.3 and $|u|<1$, we have

$$
\operatorname{PCBI}\left(I, u, q^{b}\right)=P\left(u, q^{b}\right) H\left(u, q^{b}\right)^{k},
$$

where $H\left(u, q^{b}\right):=b P\left(u, q^{b}\right)^{-b}(1-u)^{-b} S\left(u, q^{b}\right)$, with $P\left(u, q^{b}\right), S\left(u, q^{b}\right)$ as in Definition 4.1.

Proof. Let $G=\operatorname{Gal}(K / F)$. By Corollary 2.6, a matrix $X_{F}$ is $f_{i}$-primary cyclic for all $i \in I$ if and only if there exists a subset $I^{\prime}=\left\{g_{1}, \ldots, g_{k}\right\} \subseteq \operatorname{Irr}\left(q^{b}, 1\right)$ with $\left|I^{\prime}\right|=k$ such that, for each $i \leq k, g_{i}$ divides $f_{i}$, the $g_{i}$-primary component of $X_{K}$ is cyclic, and for $1 \neq \sigma \in G, g_{i}^{\sigma}$ does not divide $m_{X, K}$. For such a subset $I^{\prime}$ and, for $h \in \operatorname{Irr}\left(q^{b}\right)$, set

$$
x_{h, \lambda}= \begin{cases}0 & \text { if } h \in I^{\prime}, \text { and either } \lambda=(), \text { or } \lambda \neq(|\lambda|, 0, \ldots) \text { with }|\lambda|>0 ; \\ 0 & \text { if for some nontrivial } \sigma \in G, h^{\sigma} \in I^{\prime} ; \\ 1 & \text { if } h \in I^{\prime}, \lambda=(|\lambda|, 0, \ldots) \text { with }|\lambda|>0, \text { and } h^{\sigma} \notin I^{\prime} \text { for } 1 \neq \sigma \in G ; \\ 1 & \text { if } h \notin \bigcup_{\sigma \in G}\left(I^{\prime}\right)^{\sigma} .\end{cases}
$$

Let $X \in \mathrm{M}\left(c, q^{b}\right)$ : then $X$ contributes 1 to the $I^{\prime}$-cycle index (3.1), evaluated at $\mathbf{x}$, if and only if, for every $g_{i} \in I^{\prime}, \lambda\left(X, g_{i}\right)=(|\lambda|, 0, \ldots)$, with $|\lambda|>0$, and $\lambda\left(X, g_{i}^{\sigma}\right)=()$ for all nontrivial $\sigma \in G$; and $X$ contributes zero otherwise. This is precisely the set of matrices which, for every $g_{i} \in I^{\prime}$ and nontrivial $\sigma$, are $g_{i}$ primary cyclic and $g_{i}^{\sigma} \nmid m_{X, K}(t)$.

Arguing as in the proof of Theorem 3.6 (and in particular noting (3.4)), the number of matrices $X$ which contribute 1 to the $I^{\prime}$-cycle index of $\mathrm{M}\left(c, q^{b}\right)$ is the same for each choice of the $k$-element set $I^{\prime}$. There are $b^{k}$ subsets $I^{\prime}$ corre- 
sponding to a given $k$-subset $I \subseteq \operatorname{Irr}(q, b)$, and by Corollary 2.6, each member of $\operatorname{pcbI}\left(I, c, q^{b}\right)$ contributes 1 for exactly one of these subsets $I^{\prime}$. Hence the number of $X \in \mathrm{M}\left(c, q^{b}\right)$ for which (3.1) evaluates to 1 with the above assignment of the $x_{h, \lambda}$ is therefore $\left|\operatorname{pcbI}\left(I, c, q^{b}\right)\right| / b^{k}$. Set $I^{*}=\bigcup_{\sigma \in G}\left(I^{\prime}\right)^{\sigma}$. Then since by Corollary 2.6 we have $g^{\sigma} \neq g$ for each $g \in I^{\prime}$ and each nontrivial $\sigma \in G$, we have $\left|I^{*}\right|=b k$. Hence, by Theorem 3.6,

$$
\begin{array}{r}
\operatorname{PCBI}\left(u, q^{b}\right)=b^{k} \prod_{h \in\left(\operatorname{Irr}\left(q^{b}\right) \backslash I^{*}\right)}\left(1+\sum_{\lambda \neq()} \frac{u^{|\lambda| \operatorname{deg} h}}{c\left(\lambda, \operatorname{deg} h, q^{b}\right)}\right) \\
\times \prod_{h \in I^{\prime}}\left(\sum_{\lambda=(|\lambda|, 0, \ldots) \neq()} \frac{u^{|\lambda| \operatorname{deg} h}}{c\left(\lambda, \operatorname{deg} h, q^{b}\right)}\right) .
\end{array}
$$

Now since every polynomial in $I^{\prime}$ is linear, and since by [7, Table 1] we have that $c\left((|\lambda|, 0, \ldots), 1, q^{b}\right)=q^{|\lambda| b}\left(1-q^{-b}\right)$, it follows that

$$
\begin{aligned}
\prod_{h \in I^{\prime}}\left(\sum_{\lambda=(|\lambda|, 0, \ldots) \neq()} \frac{u^{|\lambda| \operatorname{deg} h}}{c\left(\lambda, \operatorname{deg} h, q^{b}\right)}\right) & =\prod_{h \in I^{\prime}}\left(\sum_{\alpha=1}^{\infty} \frac{u^{\alpha}}{q^{\alpha b}\left(1-q^{-b}\right)}\right) \\
& =S\left(u, q^{b}\right)^{k} .
\end{aligned}
$$

Then by Definition 4.1 and Lemma 4.2, and since $\left|I^{*}\right|=b k$,

$$
\begin{aligned}
& \operatorname{PCBI}\left(u, q^{b}\right) \\
& =b^{k} S\left(u, q^{b}\right)^{k}\left(\prod_{h \in\left(\operatorname{Irr}\left(q^{b}\right) \backslash I^{*}\right)} G\left(u^{\operatorname{deg} h}, q^{b}, \operatorname{deg} h\right)\right) \\
& =b^{k} S\left(u, q^{b}\right)^{k}\left(\prod_{h \in \operatorname{Irr}\left(q^{b}\right)} G\left(u^{\operatorname{deg} h}, q^{b}, \operatorname{deg} h\right)\right)\left(\prod_{h \in I^{*}} G\left(u, q^{b}, 1\right)\right)^{-1} \\
& =b^{k} S\left(u, q^{b}\right)^{k} P\left(u, q^{b}\right) P\left(u q^{-b}, q^{b}\right)^{-b k} \\
& =b^{k} S\left(u, q^{b}\right)^{k} P\left(u, q^{b}\right)\left((1-u) P\left(u, q^{b}\right)\right)^{-b k} \\
& =P\left(u, q^{b}\right)\left(b S\left(u, q^{b}\right)(1-u)^{-b} P\left(u, q^{b}\right)^{-b}\right)^{k}
\end{aligned}
$$

and the result follows.

\section{Combining results}

The function $\operatorname{PCBI}\left(I, u, q^{b}\right)$ counts the number of elements of $\mathrm{M}\left(c, q^{b}\right)$ which are $f$-primary cyclic (when viewed as elements of the larger algebra $\mathrm{M}(b c, q)$ ) for all the irreducibles $f$ in the $k$-subset $I \subseteq \operatorname{Irr}(q, b)$. We seek the proportion 
of matrices which are $f$-primary cyclic for some $f \in \operatorname{Irr}(q, b)$. The inclusionexclusion principle yields the following:

Theorem 5.1. For any $q, b$, let $H\left(u, q^{b}\right)=b P\left(u, q^{b}\right)^{-b}(1-u)^{-b} S\left(u, q^{b}\right)$, where $S\left(u, q^{b}\right), P\left(u, q^{b}\right)$ are as in Definition 4.1, and let $N=|\operatorname{Irr}(q, b)|$. Then

$$
\operatorname{PCB}\left(u, q^{b}\right)=P\left(u, q^{b}\right)\left(1-\left(1-H\left(u, q^{b}\right)^{N}\right) .\right.
$$

Proof. Any $X \in \mathrm{M}\left(c, q^{b}\right)$ which is primary cyclic as an element of $\mathrm{M}(n, q)$, relative to some element of $\operatorname{Irr}(q, b)$, lies in $\operatorname{pcbI}\left(I, c, q^{b}\right)$ for at least one nonempty subset $I$ of $\operatorname{Irr}(q, b)$. Thus for every positive integer $c$,

$$
\operatorname{pcb}\left(c, q^{b}\right)=\bigcup_{\substack{I \subseteq \operatorname{Irr}(q, b) \\ I \neq \emptyset}} \operatorname{pcbI}\left(I, c, q^{b}\right)
$$

and by the inclusion-exclusion principle, setting $N=|\operatorname{Irr}(q, b)|$,

$$
\left|\operatorname{pcb}\left(c, q^{b}\right)\right|=\sum_{i=1}^{N}(-1)^{i+1}\left(\sum_{I \subseteq \operatorname{Irr}(q, b),|I|=i}\left|\operatorname{pcbI}\left(I, c, q^{b}\right)\right|\right) .
$$

By Lemma 4.4, the value of $\left|\operatorname{pcbI}\left(I, c, q^{b}\right)\right|$ depends only on $|I|$. Thus choosing an $i$-element subset $I_{i}$ of $\operatorname{Irr}(q, b)$, we have

$$
\sum_{I \subseteq \operatorname{Irr}(q, b),|I|=i}\left|\operatorname{pcbI}\left(I, c, q^{b}\right)\right|=\left(\begin{array}{c}
N \\
i
\end{array}\right)\left|\operatorname{pcbI}\left(I_{i}, c, q^{b}\right)\right| .
$$

Hence

$$
\left|\operatorname{pcb}\left(c, q^{b}\right)\right|=\sum_{i=1}^{N}(-1)^{i+1}\left(\begin{array}{c}
N \\
i
\end{array}\right)\left|\operatorname{pcbI}\left(I_{i}, c, q^{b}\right)\right|,
$$

and a similar relationship holds for the generating functions:

$$
\operatorname{PCB}\left(u, q^{b}\right)=\sum_{i=1}^{N}(-1)^{i+1}\left(\begin{array}{c}
N \\
i
\end{array}\right)\left|\operatorname{PCBI}\left(I_{i}, u, q^{b}\right)\right| .
$$

Now by Lemma 4.4, writing $P=P\left(u, q^{b}\right)$ and $H=H\left(u, q^{b}\right)$, we have

$$
\begin{aligned}
\operatorname{PCB}\left(u, q^{b}\right) & =P\left(\sum_{i=1}^{N}(-1)^{i+1}\left(\begin{array}{c}
N \\
i
\end{array}\right) P H^{i}\right) \\
& =P\left(1-\sum_{i=0}^{N}(-1)^{i}\left(\begin{array}{c}
N \\
i
\end{array}\right) H^{i}\right) \\
& =P\left(1-(1-H)^{N}\right) .
\end{aligned}
$$




\begin{tabular}{ll}
\hline $\boldsymbol{c}$ & $\boldsymbol{P}_{\boldsymbol{M}}\left(\boldsymbol{c}, \boldsymbol{q}^{\boldsymbol{b}}\right)$ \\
\hline 1 & $1-q q^{-b}$ \\
\hline 2 & $\frac{1}{2}+\left(\frac{3}{2}-\frac{b}{2}\right) q^{-b}+\left(-\frac{b}{2}-q+\frac{b q}{2}-\frac{q^{2}}{2}\right) q^{-2 b}$ \\
& $+\left(-1+\frac{b q}{2}-\frac{q^{2}}{2}\right) q^{-3 b}+q q^{-4 b}$ \\
\hline $3 \quad \frac{2}{3}$ & $+\left(\frac{1}{3}-\frac{q}{2}\right) q^{-b}+\left(\frac{4}{3}-\frac{b}{2}-\frac{b^{2}}{6}+q-\frac{b q}{2}\right) q^{-2 b}$ \\
& $+\left(-\frac{1}{3}-\frac{b^{2}}{3}-\frac{b q}{2}+\frac{b^{2} q}{6}-q^{2}+\frac{b q^{2}}{2}-\frac{q^{3}}{6}\right) q^{-3 b}$ \\
& $+\left(-1-\frac{b^{2}}{3}+\frac{q}{2}-b q+\frac{b^{2} q}{3}-q^{2}+b q^{2}-\frac{q^{3}}{3}\right) q^{-4 b}$ \\
& $+\left(-1+\frac{b}{2}-\frac{b^{2}}{6}-\frac{b q}{2}+\frac{b^{2} q}{3}+b q^{2}-\frac{q^{3}}{3}\right) q^{-5 b}$ \\
& $+\left(-\frac{b q}{2}+\frac{b^{2} q}{6}+q^{2}+\frac{b q^{2}}{2}-\frac{q^{3}}{6}\right) q^{-6 b}+\left(1+q^{2}\right) q^{-7 b}-q q^{-8 b}$ \\
\hline
\end{tabular}

Table 1 . The proportion $P_{M}\left(c, q^{b}\right)$ of $f$-primary cyclic matrices in a subalgebra $M\left(c, q^{b}\right)$ of $M(b c, q)$, relative to some $f \in \operatorname{Irr}(q, b)$. As $q^{b}$ grows, $P_{M}\left(c, q^{b}\right)$ rapidly approaches a positive constant.

Theorem 5.1 shows us how to compute easily (using, e.g., Mathematica [17]) the Taylor coefficients of $\operatorname{PCB}\left(u, q^{b}\right)$, and hence values of $\left|\operatorname{pcb}\left(c, q^{b}\right)\right| /\left|\mathrm{M}\left(c, q^{b}\right)\right|$ for small $c$. We summarise some small cases in Table 1 . The data suggests that the proportion has a nonzero constant term. If this were true in general, then for every triple $(c, q, b)$ the proportion would be nontrivial. We use methods from complex analysis to examine the asymptotic behaviour as $c \rightarrow \infty$. The following fact can be found, for example, in [6, Lemma 1.3.3].

Lemma 5.2. Suppose that $g(u)=\sum a_{n} u^{n}$ and $g(u)=f(u) /(1-u)$ for $|u|<1$. If $f(u)$ is analytic with a radius of convergence $R>1$, then $a_{n} \rightarrow f(1)$ as $n \rightarrow \infty$, and $\left|a_{n}-f(1)\right|=O\left(d^{-n}\right)$ for any $d<R$.

We apply this lemma to $\operatorname{PCB}\left(u, q^{b}\right)$ to obtain one of our main results:

Proof of Theorem 1.1 (i). By Lemma 5.1, writing $N=|\operatorname{Irr}(q, b)|=N(q, b)$,

$$
\operatorname{PCB}\left(u, q^{b}\right)=P\left(u, q^{b}\right)\left(1-\left(1-H\left(u, q^{b}\right)\right)^{N}\right) .
$$

Set $L\left(u, q^{b}\right)=(1-u) \operatorname{PCB}\left(u, q^{b}\right)$. By (4.3) and Definition 4.1 we have

$$
L\left(u, q^{b}\right)=\omega\left(1, q^{b}\right)^{-1}\left(1-\left(1-H\left(u, q^{b}\right)\right)^{N}\right) .
$$

Now by Lemma 4.2, writing $S=S\left(u, q^{b}\right)$ and $P=P\left(u, q^{b}\right)$ for brevity,

$$
H\left(u, q^{b}\right)=b P^{-b}(1-u)^{-b} S=\frac{b}{q^{b}-1} \frac{u}{1-u q^{-b}} \prod_{i=1}^{\infty}\left(1-u q^{-b i}\right)^{b}
$$


which converges for all $|u|<q^{b}$. In particular, $H\left(1, q^{b}\right)$ exists and satisfies

$$
H\left(1, q^{b}\right)=\frac{b q^{-b}}{\left(1-q^{-b}\right)^{2}} \omega\left(1, q^{b}\right)^{b} .
$$

It follows that

$$
L\left(1, q^{b}\right)=\omega\left(1, q^{b}\right)^{-1}\left(1-\left(1-H\left(1, q^{b}\right)\right)^{N}\right) .
$$

By Lemma 5.2, we have $\lim _{c \rightarrow \infty}\left|\operatorname{pcb}\left(c, q^{b}\right)\right| /\left|\operatorname{GL}\left(c, q^{b}\right)\right|=L\left(1, q^{b}\right)$, and so

$$
\begin{aligned}
P_{M}\left(\infty, q^{b}\right) & =\lim _{c \rightarrow \infty} \frac{\left|\operatorname{pcb}\left(c, q^{b}\right)\right|}{\left|\mathrm{M}\left(c, q^{b}\right)\right|} \\
& =\omega\left(1, q^{b}\right) \lim _{c \rightarrow \infty} \frac{\left|\operatorname{pcb}\left(c, q^{b}\right)\right|}{\left|\operatorname{GL}\left(c, q^{b}\right)\right|} \\
& =1-\left(1-H\left(1, q^{b}\right)\right)^{N} .
\end{aligned}
$$

Theorem 1.1 (i) is proved.

The following lemma is used to study the asymptotics of $P_{M}\left(\infty, q^{b}\right)$ as $q^{b}$ grows:

Lemma 5.3. (i) If $x \in\left[0, \frac{1}{3}\right)$, then $\prod_{i=1}^{\infty}\left(1-x^{i}\right)>1-x-x^{2}>\frac{5}{9}$.

(ii) If $x \in\left[0, \frac{1}{2}\right]$ and $b$ is a positive integer, then $1-2 b x \leq\left(1-x-x^{2}\right)^{b}$.

(iii) If $x>1$, then $\frac{x}{\log x}>x^{1 / 2}$.

(iv) If $x \in\left(0, \frac{1}{2}\right)$, then $\frac{1}{1-x}<1+x+2 x^{2}$.

Proof. (i) This is proved in [13, Lemma 3.5].

(ii) We prove this inductively on $b$. For $b=1$ the inequality holds since $0 \leq$ $x<1$. Suppose that $b \geq 1$ and $1-2 b x \leq\left(1-x-x^{2}\right)^{b}$. Then

$\left(1-x-x^{2}\right)^{b+1} \geq\left(1-x-x^{2}\right)(1-2 b x)=1-(2 b+1) x+(2 b-1) x^{2}-2 b x^{3}$,

and this is at least $1-2(b+1) x-x^{2}$ since $2 b x^{2}(1-x) \geq 0$. Then since $0 \leq$ $x \leq \frac{1}{2}$, we have $-x^{2} \geq-x$, which yields the required inequality, and hence the result is proved by induction.

(iii) Since $x>1$, the required inequality is equivalent to $x^{1 / 2}>\log x$. Examining the derivative of $f(x):=x^{1 / 2}-\log x$, we see that, for $x>1, f(x)$ has a unique minimum at $x=4$. Then since $f(4)>0$, it follows that $f(x)>0$ for all $x>1$. 
(iv) Let $f(x)=\left(1+x+2 x^{2}\right)(1-x)$. The required inequality holds if and only if $f(x)>1$ (since $x \in\left(0, \frac{1}{2}\right)$ ). On multiplying we find $f(x)=1+x^{2}-2 x^{3}$ and this is greater than 1 since $x^{2}-2 x^{3}=x^{2}(1-2 x)>0$.

Lemma 5.4. Let $t \geq 1, \epsilon \in(0,1)$, and suppose that $c>\max \left\{1,\left(\frac{t}{\log (1-\epsilon)}\right)^{2}\right\}$. Then

$$
c^{t} \leq(1-\epsilon)^{-c} .
$$

Proof. The inequality is equivalent to $t \log c \leq-c \log (1-\epsilon)$. Since $\log c>0$, and since $0<1-\epsilon<1$ implies $\log (1-\epsilon)<0$, this holds if and only if

$$
-\frac{t}{\log (1-\epsilon)} \leq \frac{c}{\log c} \text {. }
$$

By Lemma 5.3 (iii), $c / \log c>c^{1 / 2}$, and by assumption $c^{1 / 2} \geq-t / \log (1-\epsilon)$, yielding inequality (5.3).

Proposition 5.5. Let $P_{M}\left(\infty, q^{b}\right)=\lim _{c \rightarrow \infty}\left|\operatorname{pcb}\left(c, q^{b}\right)\right| /\left|\mathrm{M}\left(c, q^{b}\right)\right|$, where $b \geq 2$ and $q^{b}>4$. Then

$$
-\frac{4 b}{e q^{b / 2}}<P_{M}\left(\infty, q^{b}\right)-\left(1-e^{-1}\right)<\frac{1+b}{e q^{b}}+\frac{2(1+b)^{2}}{e q^{2 b}},
$$

so that

$$
\left|P_{M}\left(\infty, q^{b}\right)-\left(1-e^{-1}\right)\right|<4 e^{-1} b q^{-b / 2} .
$$

Proof. By Theorem 1.1 (i), $P_{M}\left(\infty, q^{b}\right)=1-\left(1-H\left(1, q^{b}\right)\right)^{N}$, with $H\left(1, q^{b}\right)$ as in (5.2) above. We consider the behaviour of $\left(1-H\left(1, q^{b}\right)\right)^{N}$ as $q$ and $b$ grow. Since $\omega\left(1, q^{b}\right)=\prod_{i=1}^{\infty}\left(1-q^{-b i}\right)$, and since $q^{-b} \leq \frac{1}{4}$, it follows from Lemma 5.3 (i) that

$$
1-q^{-b}-q^{-2 b}<\omega\left(1, q^{b}\right)<1-q^{-b} .
$$

Applying Lemma 5.3 (ii) with $x=q^{-b}$ gives

$$
1-2 b q^{-b}<\omega\left(1, q^{b}\right)^{b}<1-q^{-b} .
$$

Now as $N:=N(q, b)=\frac{1}{b} \sum_{d \mid b} \mu(d) q^{d / b}$, we have

$$
\frac{1}{b}\left(q^{b}-2 q^{b / 2}\right) \leq N(q, b) \leq \frac{q^{b}}{b} .
$$

Thus

$$
\left(1-H\left(1, q^{b}\right)\right)^{(1 / b) q^{b}} \leq\left(1-H\left(1, q^{b}\right)\right)^{N} \leq\left(1-H\left(1, q^{b}\right)\right)^{(1 / b)\left(q^{b}-2 q^{b / 2}\right)},
$$


and so (with $H$ denoting $H\left(1, q^{b}\right)$ for simplicity):

$$
\frac{q^{b}}{b} \log (1-H) \leq N \log (1-H) \leq \frac{1}{b}\left(q^{b}-2 q^{b / 2}\right) \log (1-H) .
$$

Using the inequality $1-\frac{1}{x} \leq \log x \leq x-1$, which holds for all $x>0$, we have

$$
\frac{q^{b}}{b} \frac{H}{H-1} \leq N \log (1-H) \leq-\frac{1}{b}\left(q^{b}-2 q^{b / 2}\right) H .
$$

Substituting for $H$ using (5.2) and rearranging gives

$$
\begin{aligned}
\frac{-\omega\left(1, q^{b}\right)^{b}}{\left(1-q^{-b}\right)^{2}-b q^{-b} \omega\left(1, q^{b}\right)^{b}} & \leq N \log (1-H) \\
& \leq-\frac{1}{b}\left(q^{b}-2 q^{b / 2}\right) \frac{b q^{-b}}{\left(1-q^{-b}\right)^{2}} \omega\left(1, q^{b}\right)^{b} .
\end{aligned}
$$

Using the right inequality of (5.4) and observing a geometric series gives

$$
\begin{aligned}
\frac{-\omega\left(1, q^{b}\right)^{b}}{\left(1-q^{-b}\right)^{2}-b q^{-b} \omega\left(1, q^{b}\right)^{b}} & >\frac{-\left(1-q^{-b}\right)}{\left(1-q^{-b}\right)^{2}-b q^{-b}\left(1-q^{-b}\right)} \\
& =\frac{-1}{1-q^{-b}-b q^{-b}} \\
& =\frac{-1}{1-(1+b) q^{-b}} .
\end{aligned}
$$

If $q^{b} \geq 9$, then applying Lemma 5.3 (iv) with $x=(1+b) q^{-b}$ gives

$$
\frac{-1}{1-(1+b) q^{-b}} \geq-1-(1+b) q^{-b}-2(1+b)^{2} q^{-2 b},
$$

and this is true also (with equality) if $q^{b}=8$. Thus for all $q^{b}>4$, we have

$$
N \log (1-H)>-1-(1+b) q^{-b}-2(1+b)^{2} q^{-2 b} .
$$

On the other hand, we have, using the left inequality in (5.4), and since $q^{b}>4$ implies

$$
\frac{1}{\left(1-q^{-b}\right)^{2}}<\frac{1}{(3 / 4)^{2}}=\frac{16}{9}<2
$$


that

$$
\begin{aligned}
-\frac{1}{b} & \left(q^{b}-2 q^{b / 2}\right) \frac{b q^{-b}}{\left(1-q^{-b}\right)^{2}} \omega\left(1, q^{b}\right)^{b} \\
& =-\left(1-2 q^{-b / 2}\right) \frac{\omega\left(1, q^{b}\right)^{b}}{\left(1-q^{-b}\right)^{2}} \\
& <\frac{-\left(1-2 q^{-b / 2}\right)\left(1-2 b q^{-b}\right)}{\left(1-q^{-b}\right)^{2}} \\
& =-1+\frac{2 q^{-b / 2}+2(b-1) q^{-b}-4 b q^{-3 b / 2}+q^{-2 b}}{\left(1-q^{-b}\right)^{2}} \\
& <-1+2\left(2 q^{-b / 2}+2(b-1) q^{-b}-4 b q^{-3 b / 2}+q^{-2 b}\right) .
\end{aligned}
$$

Since $-4 b q^{-3 b / 2}$ is negative, and $2 q^{-b}>q^{-2 b}$, this is less than $-1+4 q^{-b / 2}+$ $4 b q^{-b}$. Thus we have proved that

$$
\begin{aligned}
-1-(1+b) q^{-b}-2(1+b)^{2} q^{-2 b} & <N \log (1-H) \\
& <-1+4 q^{-b / 2}+4 b q^{-b},
\end{aligned}
$$

and so exponentiating,

$$
\begin{aligned}
\exp \left(-1-(1+b) q^{-b}-2(1+b)^{2} q^{-2 b}\right) & <(1-H)^{N} \\
& <\exp \left(-1+4 q^{-b / 2}+4 b q^{-b}\right) .
\end{aligned}
$$

Now for $0 \leq x \leq 1$ we have $e^{x} \leq 1+x+\frac{3}{4} x^{2}$ and $e^{-x}>1-x$ (see for example [8, Lemma 2.3]). The first inequality implies that

$$
\begin{aligned}
(1-H)^{N}< & e^{-1}\left(1+4 q^{-b / 2}+4 b q^{-b}+\frac{3}{4}\left(4 q^{-b / 2}+4 b q^{-b}\right)^{2}\right) \\
= & e^{-1}+4 e^{-1} q^{-b / 2}+4 e^{-1}(b+3) q^{-b}+24 e^{-1} b q^{-3 b / 2} \\
& \quad+12 e^{-1} b^{2} q^{-2 b} \\
& <e^{-1}+4 b e^{-1} q^{-b / 2}
\end{aligned}
$$

and the second inequality gives

$$
\begin{aligned}
(1-H)^{N} & >e^{-1}\left(1-(1+b) q^{-b}-2(1+b)^{2} q^{-2 b}\right) \\
& =e^{-1}-e^{-1}(1+b) q^{-b}-2 e^{-1}(1+b)^{2} q^{-2 b} .
\end{aligned}
$$

Recalling that $P_{M}\left(\infty, q^{b}\right)=1-(1-H)^{N}$, the first inequality in the statement is proved by subtracting these two values from 1 . The second inequality follows immediately from the first. 


\subsection{Proof of Theorem 1.1 (ii)}

Finally, we apply the method of Wall (see [6]) to $\mathrm{M}\left(c, q^{b}\right)$ to prove the second part of our main result, which gives a useful lower bound on $\left|\operatorname{pcb}\left(c, q^{b}\right)\right| /\left|\mathrm{M}\left(c, q^{b}\right)\right|$ for sufficiently large $c$. The inequality we require is proved in Proposition 5.10, thus completing the proof of Theorem 1.1. We introduce the following notation, following Fulman in [5]: for a function $X(u)$ of a complex variable, we denote by $\left[u^{c}\right] X$ the coefficient of $u^{c}$ in the Maclaurin series of $X$.

Lemma 5.6. Let $X(u)$ be an analytic function of a complex variable, and let $t$ be a positive integer.

(i) For all $c \geq 1$, we have

$$
\left[u^{c}\right]\left(\frac{X(u)}{1-u}\right)=\sum_{i=0}^{c}\left[u^{i}\right] X(u) .
$$

(ii) Suppose there exist constants $a_{1}, a_{2}$ such that $\left|\left[u^{c}\right] X(u)\right| \leq a_{1} a_{2}^{-c}$, for all $c \geq 0$. Then for all $c \geq 0$, we have

$$
\left|\left[u^{c}\right]\left(X(u)^{t}\right)\right| \leq a_{1}^{t}(c+1)^{t-1} a_{2}^{-c} .
$$

Proof. (i) Let $x_{i}:=\left[u^{i}\right] X(u)$. Then

$$
\begin{aligned}
\frac{X(u)}{1-u} & =\left(x_{0}+x_{1} u+\cdots\right)\left(1+u+u^{2}+\cdots\right) \\
& =x_{0}+\left(x_{0}+x_{1}\right) u+\left(x_{0}+x_{1}+x_{2}\right) u^{2}+\cdots
\end{aligned}
$$

and (i) follows.

(ii) We proceed by induction on $t$. The result holds for $t=1$ by assumption. Let $x_{i j}:=\left[u^{j}\right] X(u)^{i}$, and suppose that $t \geq 2$ and that part (ii) holds for $X(u)^{t-1}$. Then

$$
\begin{aligned}
X(u)^{t} & =X(u)^{t-1} X(u) \\
& =\left(x_{t-1,0}+x_{t-1,1} u+\cdots\right)\left(x_{10}+x_{11} u+\cdots\right) \\
& =\sum_{c=0}^{\infty} \sum_{i=0}^{c}\left(x_{t-1, i}\right)\left(x_{1, c-i}\right) u^{c},
\end{aligned}
$$


and so by induction

$$
\begin{aligned}
\left|\left[u^{c}\right] X(u)^{t}\right| & =\left|\sum_{i=0}^{c} x_{t-1, i} x_{1, c-i}\right| \\
& \leq \sum_{i=0}^{c}\left(a_{1}^{t-1}(i+1)^{t-2} a_{2}^{-i}\right) \cdot\left(a_{1} a_{2}^{-(c-i)}\right) \\
& =a_{1}^{t} \sum_{i=0}^{c}\left((i+1)^{t-2} a_{2}^{-c}\right) \\
& \leq a_{1}^{t}(c+1)^{t-1} a_{2}^{-c},
\end{aligned}
$$

since $\sum_{j=1}^{c+1} j^{t-2} \leq(c+1)^{t-1}$. The result now follows by induction.

Lemma 5.7. Let $J\left(u, q^{b}\right)=\left(1-u q^{b}\right) \operatorname{PCB}\left(u q^{b}, q^{b}\right)$. Then for $c \geq 2$, we have

$$
\left[u^{c}\right] J\left(u, q^{b}\right)=\left(\frac{\left|\operatorname{pcb}\left(c, q^{b}\right)\right|}{\left|\mathrm{M}\left(c, q^{b}\right)\right|}-\frac{\left|\operatorname{pcb}\left(c-1, q^{b}\right)\right|}{\left|\mathrm{M}\left(c-1, q^{b}\right)\right|}\right) q^{b c} .
$$

Proof. By definition of $J\left(u, q^{b}\right)$ we have

$$
\begin{aligned}
J\left(u, q^{b}\right) & =\left(1-u q^{b}\right) \sum_{c=1}^{\infty} \frac{\left|\operatorname{pcb}\left(c, q^{b}\right)\right|}{\left|\mathrm{M}\left(c, q^{b}\right)\right|}\left(u q^{b}\right)^{c} \\
& =\frac{\left|\mathrm{pcb}\left(1, q^{b}\right)\right|}{\left|\mathrm{M}\left(1, q^{b}\right)\right|} u q^{b}+\sum_{c=2}^{\infty}\left(\frac{\left|\operatorname{pcb}\left(c, q^{b}\right)\right|}{\left|\mathrm{M}\left(c, q^{b}\right)\right|}-\frac{\operatorname{pcb}\left(c-1, q^{b}\right)}{\left|\mathrm{M}\left(c-1, q^{b}\right)\right|}\right) q^{b c} u^{c},
\end{aligned}
$$

which completes the proof.

The remainder of this section is devoted to finding an upper bound on the coefficient $\left[u^{c}\right] J\left(u, q^{b}\right)$, and using this to prove Theorem 1.1 (ii).

Lemma 5.8. Define $L\left(u, q^{b}\right):=\prod_{i=1}^{\infty}\left(1-u q^{-b i}\right)=\left(P\left(u, q^{b}\right)(1-u)\right)^{-1}$, and suppose $b>1$. Then

$$
L\left(u, q^{b}\right)=\frac{1}{1-u}\left(1+\sum_{c=1}^{\infty} \frac{(-1)^{c} q^{b c} u^{c}}{\prod_{i=1}^{c}\left(q^{b i}-1\right)}\right)
$$

and for all $c \geq 1$, we have

$$
\left|\left[u^{c}\right] L\left(u, q^{b}\right)\right| \leq a_{L} q^{-b c},
$$

where $a_{L}=2 q^{b}$. 
Proof. The first assertion follows from [1, Corollary 2.2]. For the second, observe that

$$
\begin{aligned}
{\left[u^{c}\right] L } & =1+\sum_{k=1}^{c} \frac{(-1)^{k} q^{b k}}{\prod_{i=1}^{k}\left(q^{b i}-1\right)} \\
& =1+\sum_{k=1}^{c}\left(\frac{(-1)^{k}\left(q^{b k}-1\right)}{\prod_{i=1}^{k}\left(q^{b i}-1\right)}+\frac{(-1)^{k}}{\prod_{i=1}^{k}\left(q^{b i}-1\right)}\right) \\
& =1+\sum_{k=1}^{c}\left(\frac{(-1)^{k}}{\prod_{i=1}^{k-1}\left(q^{b i}-1\right)}+\frac{(-1)^{k}}{\prod_{i=1}^{k}\left(q^{b i}-1\right)}\right) \\
& =1-1+\frac{(-1)^{c}}{\prod_{i=1}^{c}\left(q^{b i}-1\right)} \\
& =\frac{(-1)^{c} q^{-b c(c-1) / 2}}{\prod_{i=1}^{c}\left(1-q^{-b i}\right)},
\end{aligned}
$$

as all but the first and last terms of the alternating sum cancel. Now for all $c$, we have both $q^{-b c(c-1)} \leq q^{b} \cdot q^{-b c}$, and

$$
\prod_{i=1}^{c}\left(1-q^{-b i}\right)>\prod_{i=1}^{\infty}\left(1-q^{-b i}\right)>\frac{1}{2}
$$

by Lemma 5.3 (i), and so $\left|\left[u^{c}\right] L\right| \leq 2 q^{b} \cdot q^{-b c}$.

Lemma 5.9. Let $J\left(u, q^{b}\right)$ be as defined in Lemma 5.7, and suppose that $b>1$. Let

$$
M_{q^{b}}=\left(\frac{\max \left\{b-1, q^{b} / b\right\}}{\log (3 / 4)}\right)^{2} .
$$

Then for $c \geq M_{q^{b}}$, and

$$
a_{\boldsymbol{J}}=\frac{8}{3}\left(\frac{b q^{b}}{q^{b}-1} 2^{b}\left(2 q^{b}\right)^{b} q^{b^{2}}\right)^{q^{b} / b}
$$

we have

$$
\left|\left[u^{c}\right] J\left(u, q^{b}\right)\right|<a_{J}
$$

and hence

$$
\left|\frac{\operatorname{pcb}\left(c+1, q^{b}\right)}{\left|\mathrm{M}\left(c+1, q^{b}\right)\right|}-\frac{\operatorname{pcb}\left(c, q^{b}\right)}{\left|\mathrm{M}\left(c, q^{b}\right)\right|}\right|<a_{J} q^{-b c} .
$$


Proof. Using Theorem 5.1, the fact that $P\left(u q^{b}, q^{b}\right)=P\left(u, q^{b}\right)\left(1-u q^{b}\right)^{-1}$, the definition of $H\left(u q^{b}, q^{b}\right)$ from the right-hand side of (5.1), and (4.3), we have (with $N=|\operatorname{Irr}(q, b)|$ )

$$
\begin{aligned}
J\left(u, q^{b}\right) & =\left(1-u q^{b}\right) P\left(u q^{b}, q^{b}\right)\left(1-\left(1-H\left(u q^{b}, q^{b}\right)\right)^{N}\right) \\
& =P\left(u, q^{b}\right)\left[1-\left(1-\frac{b q^{b}}{q^{b}-1} \frac{u}{1-u} \prod_{i=1}^{\infty}\left(1-u q^{b-b i}\right)^{b}\right)^{N}\right] \\
& =P\left(u, q^{b}\right)\left[1-\left(1-\frac{b q^{b}}{q^{b}-1} \frac{u}{1-u} \prod_{i=0}^{\infty}\left(1-u q^{-b i}\right)^{b}\right)^{N}\right] \\
& =P\left(u, q^{b}\right)\left[1-\left(1-\frac{b q^{b}}{q^{b}-1} \frac{u}{1-u} P\left(u, q^{b}\right)^{-b}\right)^{N}\right] \\
& =P\left(u, q^{b}\right)\left[1-\left(1-\frac{b q^{b}}{q^{b}-1} u(1-u)^{b-1} L\left(u, q^{b}\right)^{b}\right)^{N}\right],
\end{aligned}
$$

since $L\left(u, q^{b}\right)=\left((1-u) P\left(u, q^{b}\right)\right)^{-1}$ by definition. By Lemma 5.8, we have $\left|\left[u^{c}\right] L\right| \leq a_{L} q^{-b c}$, where $a_{L}=2 q^{b}$, and hence by Lemma $5.6(\mathrm{ii}),\left|\left[u^{c}\right] L^{b}\right|$ is bounded above by $a_{L}^{b}(c+1)^{b-1} q^{-b c}$. Then

$$
\begin{aligned}
\left|\left[u^{c}\right]\left((1-u)^{b-1} L^{b}\right)\right| & \leq \sum_{k=0}^{b}\left(\begin{array}{l}
b \\
k
\end{array}\right) a_{L}^{b}(c-k+1)^{b-1} q^{-b(c-k)} \\
& <\sum_{k=0}^{b}\left(\begin{array}{l}
b \\
k
\end{array}\right) a_{L}^{b}(c+1)^{b-1} q^{-b(c-b)} \\
& =a_{L}^{b}(c+1)^{b-1} q^{-b(c-b)}\left(\sum_{k=0}^{b}\left(\begin{array}{l}
b \\
k
\end{array}\right)\right) \\
& =2^{b} a_{L}^{b} q^{b^{2}}(c+1)^{b-1} q^{-b c}
\end{aligned}
$$

Multiplication by $u$ 'shifts' the coefficients, so that $c$ is replaced with $c-1$ : that is,

$$
\left|\left[u^{c}\right]\left(u(1-u)^{b-1} L\left(u, q^{b}\right)^{b}\right)\right|<2^{b} a_{L}^{b} q^{b^{2}+b} c^{b-1} q^{-b c} .
$$

It follows that

$$
\left|\left[u^{c}\right]\left(\frac{b q^{b}}{q^{b}-1} u(1-u)^{b-1} L\left(u, q^{b}\right)^{b}\right)\right|<\frac{b q^{2 b}}{q^{b}-1} 2^{b} a_{L}^{b} q^{b^{2}} c^{b-1} q^{-b c},
$$


and since subtracting the function from 1 has no effect on the absolute value of any coefficients when $c \geq 1$, we have (for $c>1$ ) that

$$
\left|\left[u^{c}\right]\left(1-\frac{b q^{b}}{q^{b}-1} u(1-u)^{b-1} L\left(u, q^{b}\right)^{b}\right)\right|<\frac{b q^{b}}{q^{b}-1} 2^{b} a_{L}^{b} q^{b^{2}} c^{b-1} q^{-b c},
$$

and so by Lemma 5.4 with $t=b-1, \epsilon=\frac{1}{4}$, we have, for $c \geq\left(\frac{b-1}{\log (3 / 4)}\right)^{2}$ (and hence $c>1$ ),

$$
\left|\left[u^{c}\right]\left(1-\frac{b q^{b}}{q^{b}-1} u(1-u)^{b-1} L\left(u, q^{b}\right)^{b}\right)\right|<\frac{b q^{b}}{q^{b}-1} 2^{b} a_{L}^{b} q^{b^{2}}\left(\frac{3 q^{b}}{4}\right)^{-c} .
$$

Again applying Lemma 5.6 (ii), with $t=N$, and since by [12], $N \leq q^{b} / b$, we have

$$
\begin{aligned}
\mid\left[u^{c}\right]( & \left.1-\frac{b q^{b}}{q^{b}-1} u(1-u)^{b-1} L\left(u, q^{b}\right)^{b}\right)^{N} \mid \\
& <\left(\frac{b q^{b}}{q^{b}-1} 2^{b} a_{L}^{b} q^{b^{2}}\right)^{q^{b} / b}(c+1)^{q^{b} / b}\left(\frac{3 q^{b}}{4}\right)^{-c} .
\end{aligned}
$$

Then setting

$$
a_{J}=\frac{8}{3}\left(\frac{b q^{b}}{q^{b}-1} 2^{b} a_{L}^{b} q^{b^{2}}\right)^{q^{b} / b}
$$

and again applying Lemma 5.4 (with $c+1$ in place of $c$ and $t=q^{b} / b$ ), we have, for $c>\left(\frac{q^{b}}{b \log (3 / 4)}\right)^{2}$, that

$$
(c+1)^{q^{b} / b}<\left(1-\frac{1}{4}\right)^{-c-1}=\frac{4}{3}\left(\frac{3}{4}\right)^{-c},
$$

and so

$$
\begin{aligned}
\left|\left[u^{c}\right]\left(1-\frac{b q^{b}}{q^{b}-1} u(1-u)^{b-1} L\left(u, q^{b}\right)^{b}\right)^{N}\right| & <\frac{3 a_{J}}{8} \cdot \frac{4}{3}\left(\frac{9 q^{b}}{16}\right)^{-c} \\
& =\frac{a_{J}}{2}\left(\frac{9 q^{b}}{16}\right)^{-c} .
\end{aligned}
$$

Now by (5.5), we may attain an expression for $J\left(u, q^{b}\right)$ by multiplying the above equation by $P\left(u, q^{b}\right)$ : doing so, and recalling that by definition

$$
\left[u^{c}\right] P\left(u, q^{b}\right)=\omega\left(c, q^{b}\right)^{-1}=\prod_{j=1}^{c}\left(1-q^{-b j}\right),
$$


gives

$$
\begin{aligned}
\left|\left[u^{c}\right] J\left(u, q^{b}\right)\right| & <\sum_{i=0}^{c} \prod_{j=i}^{c}\left(1-q^{-b j}\right) \frac{a_{J}}{2}\left(\frac{9 q^{b}}{16}\right)^{-i} \\
& <\frac{a_{J}}{2}\left(\sum_{i=0}^{c}\left(\frac{9 q^{b}}{16}\right)^{-i}\right)<a_{J},
\end{aligned}
$$

since $\sum_{i=0}^{c}\left(9 q^{b} / 16\right)^{-i}<2$ when $q^{b} \geq 4$.

The second assertion follows directly from Lemma 5.7.

Proposition 5.10. Suppose $b \geq 2$, and let $a_{J}, M_{q^{b}}$ be as defined in Lemma 5.9. Then for $c>M_{q^{b}}$,

$$
\begin{aligned}
\left|P_{M}\left(c, q^{b}\right)-P_{M}\left(\infty, q^{b}\right)\right| & =\left|\frac{\left|\operatorname{pcb}\left(c, q^{b}\right)\right|}{\left|\mathrm{M}\left(c, q^{b}\right)\right|}-\lim _{n \rightarrow \infty} \frac{\left|\operatorname{pcb}\left(c^{\prime}, q^{b}\right)\right|}{\left|\mathrm{M}\left(c^{\prime}, q^{b}\right)\right|}\right| \\
& \leq \frac{a_{J}}{1-q^{-b}} q^{-b c} .
\end{aligned}
$$

In particular, Theorem 1.1 (ii) holds.

Proof. By Lemma 5.9, we have

$$
\left|\frac{\operatorname{pcb}\left(c+1, q^{b}\right)}{\left|\mathrm{M}\left(c+1, q^{b}\right)\right|}-\frac{\operatorname{pcb}\left(c, q^{b}\right)}{\left|\mathrm{M}\left(c, q^{b}\right)\right|}\right|<a_{J} q^{-b c},
$$

and so for every $c^{\prime}>c>M_{q^{b}}$ we have

$$
\begin{aligned}
\left|\frac{\left|\mathrm{pcb}\left(c^{\prime}, q^{b}\right)\right|}{\left|\mathrm{M}\left(c^{\prime}, q^{b}\right)\right|}-\frac{\left|\mathrm{pcb}\left(c, q^{b}\right)\right|}{\left|\mathrm{M}\left(c, q^{b}\right)\right|}\right| & \leq \sum_{m=c}^{c^{\prime}-1}\left|\frac{\left|\mathrm{pcb}\left(m+1, q^{b}\right)\right|}{\left|\mathrm{M}\left(m+1, q^{b}\right)\right|}-\frac{\left|\mathrm{pcb}\left(m, q^{b}\right)\right|}{\left|\mathrm{M}\left(m, q^{b}\right)\right|}\right| \\
& <\sum_{m=c}^{c^{\prime}-1} a_{J} q^{-b m} \\
& =q^{-b c} a_{J}\left(\sum_{m=0}^{c^{\prime}-c-1} q^{-b m}\right) \\
& <q^{-b c} a_{J}\left(\sum_{m=0}^{\infty} q^{-b m}\right) \\
& =q^{-b c} a_{J}\left(\frac{1}{1-q^{-b}}\right) .
\end{aligned}
$$




\section{Bibliography}

[1] G. E. Andrews, The Theory of Partitions, Cambridge University Press, Cambridge, 1998.

[2] R. W. Carter, Finite Groups of Lie Type: Conjugacy Classes and Complex Characters, John Wiley \& Sons, New York, 1993.

[3] B. P. Corr, T. Popiel and C. E. Praeger, Nilpotent-independent sets and estimation in matrix algebras, LMS J. Comput. Math. 18 (2015), 404-418.

[4] D. S. Dummit and R. M. Foote, Abstract Algebra, Prentice Hall, New Jersey, 1999.

[5] J. E. Fulman, Probability in the classical groups over finite fields: Symmetric functions, stochastic algorithms, and cycle indices, Ph.D. thesis, Harvard University, Cambridge, 1997.

[6] J. Fulman, P. M. Neumann and C. E. Praeger, A generating function approach to the enumeration of matrices in classical groups over finite fields, Mem. Amer. Math. Soc. 176 (2005), no. 830, 1-90.

[7] S. P. Glasby and C. E. Praeger, Towards an efficient Meat-Axe algorithm using $f$ cyclic matrices: The density of uncyclic matrices in $M(n, q)$, J. Algebra 322 (2009), no. 3, 766-790.

[8] S. Guest and C. E. Praeger, Proportions of elements with given 2-part order in finite classical groups of odd characteristic, J. Algebra 372 (2012), 637-660.

[9] B. Hartley and T. O. Hawkes, Rings, Modules and Linear Algebra, Chapman \& Hall, London, 1980.

[10] D. F. Holt and S. Rees, Testing modules for irreducibility, J. Aust. Math. Soc. Ser. A 57 (1994), no. 1, 1-16.

[11] J. P. S. Kung, The cycle structure of a linear transformation over a finite field, Linear Algebra Appl. 36 (1981), 141-155.

[12] R. Lidl and H. Niederreiter, Finite Fields, 2nd ed., Encyclopedia Math. Appl. 20, Cambridge University Press, Cambridge, 1997.

[13] P. M. Neumann and C. E. Praeger, Cyclic matrices over finite fields, J. Lond. Math. Soc. (2) 52 (1995), no. 2, 263-284.

[14] R. A. Parker, The computer calculation of modular characters (the meat-axe), in: Computational group theory (Durham 1982), Academic Press, London (1984), 267274.

[15] G. Pólya and R. Read, Combinatorial Enumeration of Groups, Graphs, and Chemical Compounds, Springer, New York, 1987.

[16] R. Stong, Some asymptotic results on finite vector spaces, Adv. in Appl. Math. 9 (1988), no. 2, 167-199.

[17] Wolfram Research Inc., Mathematica 8.0, Champaign, Illinois, 2010. 
Received November 22, 2017; revised March 29, 2018.

\section{Author information}

Brian P. Corr, Centre for Mathematics of Symmetry and Computation, School of Mathematics and Statistics, The University of Western Australia, Crawley, WA 6009, Australia.

E-mail: brian.p.corr@gmail.com

Cheryl E. Praeger, Centre for Mathematics of Symmetry and Computation, School of Mathematics and Statistics, The University of Western Australia, Crawley, WA 6009, Australia.

E-mail: cheryl.praeger@uwa.edu.au 